\title{
Risks of complications by attending physicians after performing nighttime procedures
}

\author{
Prosanto K. Chaudhury, MD \\ Garth L. Warnock, MD \\ Thomas V. Whalen, MD \\ for the Members of the \\ Evidence Based Reviews \\ in Surgery Group* \\ *The CAGS/ACS Evidence Based \\ Reviews in Surgery Group comprises \\ Drs. N.N. Baxter, K.J. Brasel, C.J. Brown, \\ P. Chaudhury, C.S. Cutter, C.M. Divino, \\ E. Dixon, L. Dubois, G.W.N. Fitzgerald, \\ H.J.A. Henteleff, A.W. Kirkpatrick, \\ S. Latosinsky, A.R. MacLean, \\ T.M. Mastracci, R.S. McLeod, \\ A.M. Morris, L.A. Neumayer, L.K. Temple \\ and Ms. M.E. McKenzie.
}

\section{Correspondence to:}

Ms. Marg McKenzie, RN

Administrative Coordinator, EBRS

Mount Sinai Hospital, L3-010

60 Murray St., PO Box 23

Toronto ON M5T 3L9

mmckenzie@mtsinai.on.ca

DOI: $10.1503 /$ cjs. 018212

\begin{abstract}
The term "evidence-based medicine" was first coined by Sackett and colleagues as "the conscientious, explicit and judicious use of current best evidence in making decisions about the care of individual patients." The key to practising evidencebased medicine is applying the best current knowledge to decisions in individual patients. Medical knowledge is continually and rapidly expanding. For clinicians to practise evidence-based medicine, they must have the skills to read and interpret the medical literature so that they can determine the validity, reliability, credibility and utility of individual articles. These skills are known as critical appraisal skills, and they require some knowledge of biostatistics, clinical epidemiology, decision analysis and economics, and clinical knowledge.

Evidence Based Reviews in Surgery (EBRS) is a program jointly sponsored by the Canadian Association of General Surgeons (CAGS) and the American College of Surgeons (ACS) and is supported by an educational grant from ETHICON and ETHICON ENDO-SURGERY, both units of Johnson \& Johnson Medical Products, a division of Johnson \& Johnson and ETHICON Inc. and ETHICON ENDO-SURGERY Inc., divisions of Johnson \& Johnson Inc. The primary objective of EBRS is to help practising surgeons improve their critical appraisal skills. During the academic year, 8 clinical articles are chosen for review and discussion. They are selected for their clinical relevance to general surgeons and because they cover a spectrum of issues important to surgeons, including causation or risk factors for disease, natural history or prognosis of disease, how to quantify disease, diagnostic tests, early diagnosis and the effectiveness of treatment. A methodological article guides the reader in critical appraisal of the clinical article. Methodological and clinical reviews of the article are performed by experts in the relevant areas and posted on the EBRS website, where they are archived indefinitely. In addition, a listserv allows participants to discuss the monthly article. Surgeons who participate in the monthly packages can obtain Royal College of Physicians and Surgeons of Canada Maintenance of Certification credits and/or continuing medical education credits for the current article only by reading the monthly articles, participating in the listserv discussion, reading the methodological and clinical reviews and completing the monthly online evaluation and multiple choice questions.

We hope readers will find EBRS useful in improving their critical appraisal skills and in keeping abreast of new developments in general surgery. Four reviews are published in condensed versions in the Canadian fournal of Surgery and 4 are published in the fournal of the American College of Surgeons. For further information about EBRS, please refer to the CAGS or ACS websites. Questions and comments can be directed to the program administrator, Marg McKenzie, at mmckenzie@mtsinai.on.ca.
\end{abstract}

\section{Reference}

1. Evidence-Based Medicine Working Group. Evidence-based medicine. 7AMA 1992;268:2420-5. 


\section{Selected Article}

Rothschild JM, Keohane CA, Rogers S, et al. Risks of complications by attending physicians after performing nighttime procedures. FAMA 2009;302:1565-72.

\section{Abstract}

Objective: To determine whether sleep opportunities for attending surgeons and obstetricians/gynecologists are associated with the risk of complications. Design: Matched retrospective cohort study of procedures performed from 1999 to 2008 by attending physicians (86 surgeons, 134 obstetricians/gynecologists) who had been in hospital performing another procedure in adult patients for at least part of the preceding night (12:00-6:00 am postnighttime procedures). Results: A total of 9191 surgical and $957 \mathrm{ob-}$ stetrical postnighttime procedures were matched with 3552 and 3945 control procedures, respectively. Complications occurred in 101 postnighttime procedures $(5.4 \%)$ and 365 control procedures (4.9\%; odds ratio [OR] 1.09, 95\% confidence interval [CI] 0.84-1.41). Complications occurred in 82 of 1317 (6.2\%) postnighttime procedures performed by surgeons who had 6 hours of sleep or fewer versus 19 of $559(3.4 \%)$ postnighttime procedures performed by surgeons who had more than 6 hours of sleep (OR 1.72, 95\% CI 1.02-2.89). Postnighttime procedures completed after working more than 12 hours $(n=958)$ compared with 12 hours or fewer $(n=918)$ had nonsignificantly higher complication rates $(6.5 \%$ v. $4.3 \%$; OR 1.47 , 95\% CI 0.96-2.27). Conclusion: Overall, procedures performed the day after attending physicians worked overnight were not associated with significantly increased complication rates, although there was an increased rate of complications for postnighttime procedures performed by physicians who had fewer than 6 hours of sleep.

\section{Commentary}

The issue of sleep deprivation among attending surgeons and surgical trainees has become a matter of national and international debate. The discussion is timely. As of July 2012, workhour restrictions are in effect in the province of Quebec, Canada. Whereas many attending physicians and surgical residents may recoil at the thought of regulation of sleep hours for all physicians, we must at least admit that the question should be asked. However, we, the profession, should and must be the ones to ask and scientifically answer the question. If we believe in patient safety as the foremost guide for what we do, then nothing less will suffice.

The study by Rothschild and colleagues ${ }^{1}$ is a matched retrospective cohort study whose goal was to determine whether sleep opportunities for attending surgeons and obstetricians/gynecologists were associated with a risk of complications.
Cohort studies are often undertaken to establish a causal association. In retrospective cohort studies, the investigator collects data from past records but does not follow patients, as is the case with prospective studies. However, at the starting point of the study explicit data for the entire cohort can be obtained and analyzed. The first objective is to establish 2 groups - exposed versus nonexposed - in the case of our selected study, patients undergoing postnighttime surgery (exposed) versus those who did not have postnighttime procedures (nonexposed). In addition, an effort was made to match cases and controls.

The study by Rothschild and colleagues ${ }^{1}$ expands our knowledge about the role of surgeons' fatigue in the occurrence of medical errors. The investigation informs this issue since it examines the risks of experienced surgeons (rather than surgical trainees) experiencing complications after periods of sleep deprivation. The authors found that surgeons who participated in emergency procedures the night before scheduled surgery did not have an overall increase in the number of complications in the scheduled procedures (OR 1.09, 95\% CI, 0.84-1.41), but an increased rate of complications was observed among surgeons who had fewer than 6 hours of sleep between completion of their last night procedure and their first scheduled procedure the next day (OR 1.72, 95\% CI 1.02-2.89).

These findings are highly relevant to surgeons whose schedules might not allow optimal rest before conducting complex scheduled procedures after having performed emergency procedures the night before. The data are also relevant to the current teaching environment where, frequently, a large number of trainees must be supervized directly and with high quality in the operating room despite fatigue and sleep deprivation among attending supervizing faculty. The data have implications for surgical specialties where subspecialization has resulted in surgeons participating in multiple and competing call schedules (e.g., acute surgery, trauma, oncology, transplantation, critical care, hepatobiliary procedures).

As acknowledged by Rothschild and colleagues, ${ }^{1}$ the study outcomes may not be generalizable to all environments since the investigation was completed in an urban, tertiary, subspecialty referral care centre where resident trainees may have added to or lessened the effects of sleep deprivation among the attending surgeons who participated. The authors also acknowledge that they could not exclude that surgeons who performed the control daytime procedures may also have been awake the night before and therefore may have experienced consequences of missed sleep opportunities. Furthermore, there may be differences among specialties, as evidenced by the absence of a significantly increased complication rate among obstetricians/ gynecologists compared with other surgical specialties. Thus, differences related to structures and processes of care in specialties may alter susceptibility to the effects of sleep deprivation, a topic which is currently under investigation 
to explain variations in complications and mortality in hospital patients. ${ }^{2}$

The selected study complements other literature that focuses on physicians-in-training assigned declining work hours in both the United States and Europe to improve patient safety and doctors' working conditions. ${ }^{3,4}$ Whereas these legislated reductions in work hours to the 80-hour work week in the United States and to fewer than 52 hours in European Union countries (decreasing to $48 \mathrm{~h}$ in 2012) may relate to trainees, the relevance to experienced surgeons is less clear. A more recent article has probed the question of whether reduction of trainees' work hours has resulted in improved outcomes for patients; ${ }^{5}$ a total of 34 published articles from the past decade were reviewed, but there was no clear indication of whether patients were harmed or whether their outcomes improved as a result of restricted work hours. As acknowledged in that study, the conclusions were limited because they were based on studies of poor quality with retrospective analyses. Only 1 randomized controlled trial was included. A problem that was acknowledged was the inability to have doctors participate in a trial where they are subjected to long work shift durations and sleep deprivation.

Owing to the nature of cohort studies, multiple biases are possible. Selection bias is defined as a systematic error in creating the intervention groups, causing them to differ with respect to prognosis. The resulting groups differ in measured or unmeasured baseline characteristics because of the way in which participants, in this case patients undergoing surgery, were selected for the study or assigned to their study groups. For instance, it is possible or perhaps even likely that a surgeon would schedule elective procedures that he or she feels are in some way "easier" or more straightforward for the days after a nighttime duty and avoid scheduling those that are subjectively more difficult. It may be extremely difficult to control for this type of bias, as it may not be objectively quantifiable and represent a surgical gestalt.

In the case of our selected article, several potential confounding variables are not characterized. These include the presence or absence of residents and their state of rest. In addition, it is impossible to determine whether the physicians, when performing regularly scheduled elective surgery, had decreased sleep opportunities (e.g., operating at another institution, up with a sick child). The judging of the preventability of complications may have been subject to reviewers' hindsight bias.

The cohorts in this study appeared to be well matched, apart from a minor $(6 \mathrm{mo})$ age difference in the obstetrician/ gynecologist group. Every postnighttime case was matched to several contemporaneous control cases performed by the same physician. Matching relied on administrative data and International Classification of Diseases (ICD)-9 coding of cases, and was therefore subject to the limitations of data quality. We are given relatively limited information about the groups of patients (control cases and postnighttime procedures). It is therefore possible that the 2 groups are inherently different with respect to confounding factors, and this may have an impact on the conclusions. This is one of the limitations of using administrative data: important clinical data that impact on outcome are often not collected or available.

A confounder is a characteristic that is related to the outcome in terms of susceptibility or prognosis and is unevenly distributed between the groups being compared. In assessing cohort studies, it is important to identify potential confounders and examine their distribution between the groups being compared. In our selected study, cases and controls were matched primarily based on ICD-9 procedure codes. Major comorbidities were compared between groups and using the Charlson score; however, other potential confounders, such as reoperation, were not specifically addressed.

The evidence from this study underscores the difficulties of clearly establishing a causal relationship between exposure and outcome. Overall the conclusion is supported by the evidence, although the subgroup analysis $(<6 \mathrm{~h}$ sleep) should be interpreted with caution.

In broader terms, the conclusions of the study need to be interpreted and applied while considering the realities of practice. If we speculate that there should be regulation or at least guidelines on work hours, how shall we effectively care for our communities? The Health Policy Research Institute of the American College of Surgeons has determined that there should be 7.53 general surgeons for each population of 100000 people. Many communities in Canada and the United States fall far short of that ratio. What further access problems will be engendered by such potential regulations? Alternatively phrased, is it better that there always be a general surgeon available who may on occasion be tired, or is it preferable to have a well-rested surgeon available only part of the time?

Competing interests: None declared.

\section{References}

1. Rothschild JM, Keohane CA, Rogers S, et al. Risks of complications by attending physicians after performing nighttime procedures. $7 A M A$ 2009;302:1565-72.

2. Ghaferi AA, Birkmeyer JD, Dimick JB. Variation in hospital mortality associated with inpatient surgery. N Engl 7 Med 2009;361:1368-75.

3. Accreditation Council for Graduate Medical Education. Report of the ACGME work group on resident duty hours. Chicago (IL): ACGME; 2002.

4. European Union. Working time directive. Official Fournal of the European Union 2003;L 299:9-19.

5. Moonesinghe SR, Lowery J, Shahi N, et al. Impact of reduction in working hours for doctors in training on postgraduate medical education and patients' outcomes: systematic review. BMF 2011;342:d1580. 